\title{
Gradhiva
}

\section{Emmanuel Grimaud, Dieux et robots. Les théâtres d'automates divins de Bombay}

Apt, L'Archange Minotaure, coll. « L'âme du monde », 2008, 168 p., illustrations

\section{Julien Bonhomme}

\section{(2) OpenEdition}

\section{Journals}

Édition électronique

URL : http://journals.openedition.org/gradhiva/1773

DOI : 10.4000/gradhiva. 1773

ISSN : 1760-849X

Éditeur

Musée du quai Branly Jacques Chirac

Édition imprimée

Date de publication : 19 mai 2010

Pagination : 222-225

ISBN : 978-2-35744-025-8

ISSN : 0764-8928

Référence électronique

Julien Bonhomme, "Emmanuel Grimaud, Dieux et robots. Les théâtres d'automates divins de Bombay », Gradhiva [En ligne], 11 | 2010, mis en ligne le 09 mai 2010, consulté le 21 septembre 2020. URL : http:// journals.openedition.org/gradhiva/1773 ; DOI : https://doi.org/10.4000/gradhiva.1773

Ce document a été généré automatiquement le 21 septembre 2020.

(c) musée du quai Branly 


\section{Emmanuel Grimaud, Dieux et robots. Les théâtres d'automates divins de Bombay}

Apt, L'Archange Minotaure, coll. « L'âme du monde », 2008, 168 p., illustrations

Julien Bonhomme

\section{DIEUX \& ROBOTS}

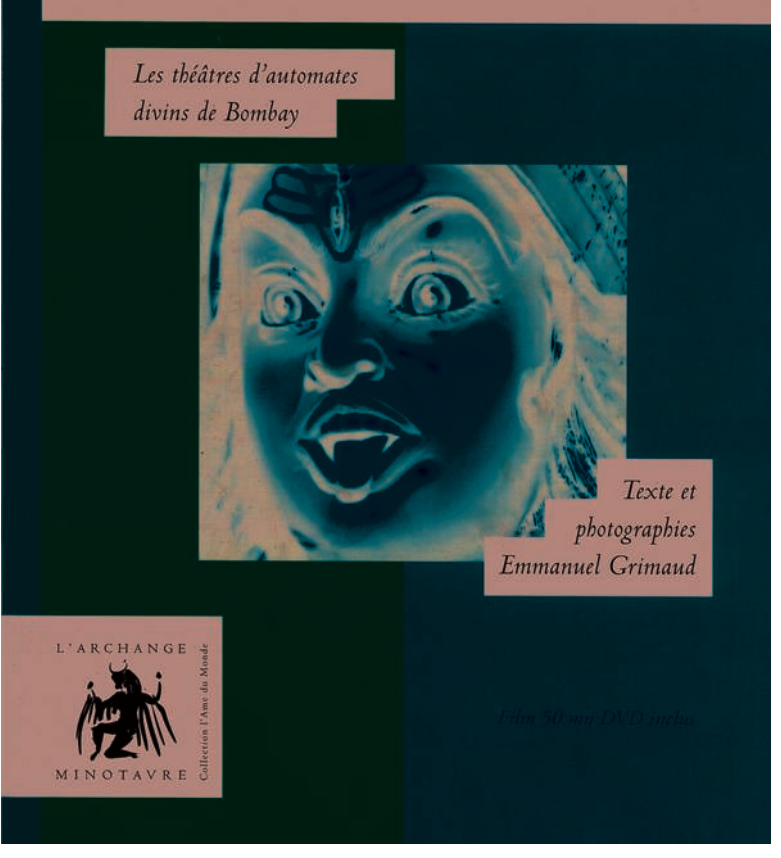

1 Comment bougent les dieux ? Tout l'intérêt du livre passionnant d'Emmanuel Grimaud consiste à aborder ce problème quelque peu abscons non pas en théologien mais, bien plus concrètement, en artisan. Il en fait ainsi une question technique plutôt que 
métaphysique: quelles poulies et quelles manivelles utiliser pour faire bouger les dieux? L'ouvrage porte en effet sur les théâtres d'automates divins en Inde : grâce à d'ingénieux procédés de mécanisation, les plateformes rituelles construites à l'occasion des fêtes religieuses deviennent de véritables spectacles animés mettant en scène les divinités du panthéon hindou. On verra par exemple la déesse Kali agiter dans sa main la tête tranchée d'un démon ou bien tirer la langue en roulant des yeux exorbités au son d'une musique de transe. Emmanuel Grimaud nous fait suivre minutieusement toutes les étapes de fabrication de ces théâtres d'automates, des premières ébauches jusqu'au spectacle final et à sa réception par le public. Mais il ne se contente pas de décrire et d'analyser, il nous donne également à voir les théâtres d'automates, leurs ateliers de construction et leur esthétique si particulière. Publié dans un grand format du type « livre d'art ${ }^{1}$ ", l'ouvrage inclut un cahier central de 120 illustrations (dont les légendes auraient gagné à ne pas être reportées en fin de volume). Il est en outre accompagné d'un film sur DVD (Cosmic City, 50 minutes). Il ne s'agit pas exactement d'un documentaire, mais plutôt d'un film expérimental déroutant qui cherche à faire éprouver au spectateur l'effet hypnotique produit par le théâtre d'automates, en utilisant pour cela tous les effets spéciaux à sa disposition (ralenti, effet stroboscopique, montage haché, bruitage, incrustation).

2 Ce travail sur les "dieux robotisés" s'inscrit dans la continuité des recherches antérieures d'Emmanuel Grimaud, qui portaient déjà sur la création et le statut des images dans l'Inde contemporaine : après un premier ouvrage sur les studios de cinéma de Bombay (Bollywood Film Studio. Paris, CNRS éditions, 2004), l'auteur a publié un petit livre sur un sosie de Gandhi pour le moins fascinant (Le Sosie de Gandhi. Paris, CNRS éditions, 2007). Ces recherches possèdent en outre une dimension collective. La rencontre troublante entre les dieux et les robots que décrit ici Emmanuel Grimaud est également au cœur des travaux de Denis Vidal, lui aussi anthropologue indianiste : dans un récent article intitulé "Anthropomorphism or sub-anthropomorphism? An anthropological approach to gods and robots " (Journal of the Royal Anthropological Institute 13[4], 2007 : 917-933), ce dernier s'interroge sur les modes de manifestation ambigus de ces entités simili-humaines. Sur le sujet, les deux auteurs ont d'ailleurs publié ensemble un catalogue d'exposition avec le roboticien japonais Tomotaka Takahashi : Vers l'infini et au-delà ! (Paris, Isthme Éditions, 2007). Ce travail participe plus largement d'un programme de recherche mené au sein d'un collectif nommé "Artmap » (dont on peut consulter le site à l'adresse suivante : http://www.artmapresearch.com). Les anthropologues, historiens et artistes de ce collectif s'intéressent aux «technologies de l'enchantement» (pour reprendre une formule d'Alfred Gell²) et se penchent sur des sujets aussi divers que les machines de spectacle, l'illusionnisme, la robotique, les combats d'animaux, l'infographie ou encore la virtuosité musicale. Le numéro 46 de la revue Terrain consacré aux «Effets spéciaux et artifices» (2006), coordonné par Emmanuel Grimaud, Sophie Houdart et Denis Vidal, donne un bon aperçu de cette recherche collective aussi originale que stimulante.

3 Le présent ouvrage d'Emmanuel Grimaud s'intéresse plus particulièrement aux plateformes rituelles créées pour la fête annuelle de Ganapati, le dieu à tête d'éléphant, à Bombay. Depuis son renouveau à la fin du XIXe siècle, ce festival religieux possède une forte dimension politique. Les manifestations publiques sont en effet l'occasion d'une intense galvanisation politique et religieuse - et les détracteurs des spectacles d'automates leur reprochent d'ailleurs de faire le jeu de la droite hindoue. Mais elles ont également une portée citoyenne : les théâtres d'automates doivent mettre en scène 
un sujet d'actualité qui relève du bien commun. Les plateformes rituelles pourront ainsi comporter, dans l'entourage de la divinité, une partie didactique évoquant la lutte contre le tabac, la corruption politique ou un nouveau projet de gare. Emmanuel Grimaud est attentif à tous ces croisements entre spectacle, religion et politique qui font la richesse des théâtres d'automates ${ }^{3}$. Il passe en revue l'ensemble des acteurs qui se mobilisent dans les associations de quartier (mandal) qui prennent en charge l'élaboration des plateformes rituelles: artisans et machinistes, mais aussi citoyens bénévoles, parrains financiers ou politiques, experts religieux ou scientifiques. Il montre ainsi comment ces acteurs sont amenés à faire de la politique au moyen des automates divins. L'auteur prend cependant soin de ne pas réduire le spectacle à un simple prétexte. Le théâtre d'automates n'est pas un support neutre qui serait cyniquement instrumentalisé par les dévots et les politiciens. En focalisant son analyse sur le travail des artisans, Emmanuel Grimaud réfléchit aux contraintes spécifiquement techniques qui rendent possible cette instrumentalisation. Il s'intéresse à la partie proprement artistique - et même artisanale - de cet « art politique » que représente le festival de Ganapati.

4 Plutôt qu'un ouvrage d'anthropologie politique et religieuse à strictement parler, Emmanuel Grimaud nous offre une véritable ethnographie des trucages (une "trucographie ", comme il l'écrit lui-même) - et c'est bien là ce qui fait la grande originalité de son travail. Il décrit en détail toute la filière des artisans et machinistes qui rivalisent d'ingéniosité pour bricoler leurs automates low-tech: on rabote le bras d'un mannequin pour lui faire adopter la pose appropriée, on tord des fils de fer ou on ajoute un ressort pour lui faire bouger la tête, on trafique tout un jeu de poulies et de cordes pour le faire voler dans les airs. L'inventivité sous contrainte de ces bricoleurs professionnels est stimulée par des moyens financiers souvent limités, par l'impossibilité de représenter le même spectacle d'une année sur l'autre ainsi que par la compétition entre associations de quartier pour créer la plateforme la plus belle ou la plus ingénieuse. Les artisans se trouvent ainsi confrontés à de multiples problèmes de synchronisation technique et de coordination humaine afin de mettre en scène ce véritable spectacle total qu'est le théâtre d'automates. En effet, une plateforme associe habituellement des formes de représentation et d'animation hétérogènes : affiches, textes, maquettes, jouets, automates, machines en tout genre, éclairages, bruitages, musique, etc. En évoquant la création des effets spéciaux dans les studios de cinéma, Emmanuel Grimaud montre comment le travail des fabricants d'automates s'inscrit dans une véritable tradition indienne $d u$ " trucage mythologique ». Le très riche corpus de films mettant en scène épisodes mythologiques et divinités hindoues témoigne bien du rapport intime entre le cinéma et l'illusionnisme (que l'on pouvait trouver chez un Méliès, par exemple). Les créateurs d'effets spéciaux bricolent toutes sortes de solutions pour réussir à donner à voir des phénomènes surnaturels. Contrairement à l'usage "scientifique» de la photographie dans le spiritisme européen ou, plus récemment, dans l'ufologie (cf. les photographies de spectres ou d'ovnis censées attester leur existence), ces trucages mythologiques ne se situent absolument pas dans une logique de la preuve : l'artifice ne doit pas passer inaperçu, mais être au contraire bien visible (en le rendant le plus goldy possible) - signe que l'on se situe dans un régime d'existence des images bien différent.

5 On ne saurait toutefois confondre les effets spéciaux du cinéma mythologique et les trucages des plateformes rituelles. Emmanuel Grimaud montre que le thêatre d'automates s'organise spécifiquement autour d'une esthétique de la lenteur. Il ne 
s'agit pas seulement d'une contrainte technique imposée par le low-tech, mais bien d'un style propre. La lenteur répétitive (les mouvements élémentaires sont indéfiniment reproduits) est censée produire sur le spectateur une " hypnose mécanique " propice à la dévotion. Le ralenti intensifie le mouvement et, ainsi, capte davantage l'attention. Ce degré supérieur de présence propre à la lenteur explique pourquoi un automate est réputé être plus efficace qu'un acteur ou un effet spécial pour donner à voir la " grâce mécanique " des dieux. Cette question de la traduction de la cosmologie dans la technologie - ce que l'auteur appelle le «design cosmologique»-donne lieu à de passionnants développements : comment traduire l'ordre divin au moyen d'artifices techniques ? L'" effet infrarouge » (une petite lumière clignotante et mobile qui se détache d'un corps pour passer à un autre) permet par exemple de donner à voir le concept de «transmigration" (samsara), central dans la cosmologie hindoue. Loin d'être neutre et indifférente, cette opération de traduction implique toujours nécessairement une invention à la fois technique et intellectuelle : les machinistes sont en effet amenés à poser des questions inédites à la théologie savante (ainsi le déconcertant : « Comment bougent les dieux?»).

Sur ce point, on pourra cependant regretter qu'il manque quand même au lecteur non spécialiste des données d'ordre général concernant le statut des divinités et de leurs représentations, les conceptions de l'ordre cosmique et du mouvement, les critères esthétiques de la grâce et de l'harmonie, la catégorisation du vivant et de l'inanimé ou encore les modalités rituelles de la dévotion. On comprend bien que l'auteur ait voulu éviter le point de vue de la théologie savante, qui pèse très lourdement dans l'indianisme classique, pour s'intéresser plutôt à la créativité en acte des fabricants d'automates - et on ne peut que souscrire entièrement à ce décalage du regard. Une information sommaire aurait cependant été la bienvenue pour saisir plus précisément comment le théâtre d'automates « met en branle » et « fait bouger » les représentations que l'on se fait habituellement des dieux et du cosmos. Par exemple, l'auteur nous en dit trop peu sur la notion de darshan (le contact visuel avec la divinité), dont on pressent pourtant qu'elle est centrale dans l'appréhension des automates divins par les spectateurs. L'auteur esquive ainsi une réflexion précise sur un certain nombre de points en se cachant parfois derrière une rhétorique «pop » (à la Gilles Deleuze ou à la Bruno Latour) un peu trop appuyée. Le statut de la description s'en trouve parfois un peu brouillé. Les notions de "vitesse hypnotique» ou de "grâce mécanique " traduisent-elles des concepts vernaculaires, ou du moins cristallisent-elles une nébuleuse de représentations indigènes? De même, d'où vient la notion de "bougé " que l'auteur place au centre de son analyse? La distinction classique entre geste et mouvement est-elle pertinente ici (ce qui pose la question du type d'agentivité - agency - prêté aux automates et aux divinités) ? On aurait enfin aimé en savoir plus sur la notion de secousse ou d'ébranlement (jhatka), qui revient plusieurs fois dans la description.

7 La théorie indigène du mouvement - et sa dimension cosmologique - aurait ainsi mérité de plus amples développements. On voit bien en effet que le rapport entre le mouvement et l'immobilité joue un rôle décisif dans le théâtre d'automates. Sur la plateforme, seuls les éléments qui entourent la divinité centrale peuvent en réalité être animés. L'idole principale doit quant à elle rester statique, car « on ne peut prendre le darshan d'une image qui bouge ", comme le suggère un spectateur. Cette immobilité est nécessaire à l'impression de plénitude immuable et de sérénité intériorisée que doit dégager la divinité, soigneusement ornée et embellie. Le spectacle doit ainsi se boucler 
sur l'image fixe de la divinité. On voit de la sorte comment les techniques d'animation sont mises au service d'une conception analogiste du monde : il s'agit de connecter les choses et les êtres en les faisant graviter autour de l'idole immobile, point fixe qui oriente tout le système. La plateforme rituelle constitue en cela un véritable microcosme.

Tout l'enjeu de l'installation est alors de réussir à intégrer intelligemment dans ce cosmos en miniature des sujets d'actualité, qu'il s'agisse de la pollution, du terrorisme ou de tout autre problème de société. Pour ce faire, la mise en scène obéit à une logique de l'accumulation maximale typique de l'analogisme. Ce principe de "congestion divine » consiste à agglomérer le plus grand nombre de choses dans un espace restreint afin de tisser des correspondances entre elles : on s'attache à « connecter le cosmos, la pollution, la nation, la famille, les bidonvilles, la corruption et les inondations, tantôt par des fils, tantôt par des glissières, des manivelles ou des machines » (p. 160). C'est ainsi qu'une plateforme rituelle représentera à la fois le cosmos en miniature, mais aussi une réduction de Bombay (pour évoquer le trafic urbain) ou du corps humain (pour évoquer la maladie). On cherche en définitive à «faire bouger les choses» dans tous les sens du terme - l'expression renvoyant à la fois à l'animation de la plateforme et à son ambition citoyenne plus générale. Se pose ainsi une question cruciale : quels éléments du décor faut-il choisir de faire bouger (et comment) pour transmettre un message ou raconter une histoire le plus efficacement possible? Au centre du dispositif, la présence de la divinité permet alors d'élever les problèmes de société à une dimension véritablement cosmologique, ce que l'auteur appelle un «cosmoproblème ». Que cette dramatisation cosmique suscite un débat public qui va dans la «bonne voie » (dharma), est la meilleure preuve que c'est bien la voix de la divinité qui parle à travers les automates.

\section{NOTES}

1. On peut d'ailleurs regretter que les finitions éditoriales de ce «beau livre » laissent parfois à désirer : il manque une relecture du manuscrit, ce qui se traduit par des coquilles, omissions et erreurs (notamment dans la bibliographie).

2. Cf. "The technology of enchantment and the enchantment of technology ", in Jeremy Coote et Anthony Shelton (éd.), Anthropology, Art and Aesthetics.Oxford, Clarendon Press, 1992 : 40-63.

3. Bel exemple d'alliance entre divertissement et dévotion ; un spectateur lui a ainsi confié : «Je suis venu prendre la vision du dieu (darshan), mais aussi voir ce qu'ils ont fait avec la voix de l'acteur Sanjay Dutt. » (p. 147) 


\section{AUTEUR}

\section{JULIEN BONHOMME}

julienbonhomme@yahoo.fr 